\title{
Experimental Analysis of Shot-Noise Suppression in InGaAs/InAlAs Recessed Planar Diodes at Room Temperature
}

\author{
O. García-Pérez, J. Mateos, S. Pérez, T. González \\ Departamento de Física Aplicada \\ Universidad de Salamanca \\ Salamanca, Spain
}

\author{
A. Westlund, J. Grahn \\ Department of Microtechnology and Nanoscience \\ Chalmers University of Technology \\ Gothenburg, Sweden
}

\begin{abstract}
In this work, current noise measurements of submicron InGaAs/InAlAs recessed planar diodes are presented. The presence of a barrier in the potential profile under the source side of the recess may affect the statistics of passage of carriers, which in turn would lead to suppressed shot noise levels. However, evidencing this noise suppression from the measurements is challenging, due to the undesired effects from the accesses and contact resistances. The presented results are provided as a starting point for a future de-embedding process able to precisely determine the actual noise properties below the recess.
\end{abstract}

Keywords-Noise measurement; shot noise; mesoscopic devices; III-V semiconductor materials.

\section{INTRODUCTION}

Beyond being an obstacle for the signal transmission in any electronic communications system, noise can also be an important source of information about the different mechanisms of electron transport present in a semiconductor device. For instance, the interactions between the carriers can regulate its propagation along the device, leading to suppressed levels of noise [1].

More concretely, Pauli principle and/or Coulomb interaction, by affecting carrier statistics, may reduce the standard full shot noise inherent to ballistic devices [2]-[3]. On the one hand, Pauli principle has influence on the injecting statistics of the carriers at the contacts. On the other hand, Coulomb interaction may affect the propagation statistics of the carriers through the device. Both effects may lead to a current spectral density $\left(S_{\mathrm{I}}\right)$ defined as

$$
S_{\mathrm{I}}=F 2 q I
$$

where $q$ is the electron charge, $I$ is the current, and $F$ is the Fano factor, which quantifies the suppression due to the two described phenomena.

Since shot noise can play an important role in submicron semiconductor devices, understanding and characterizing the mechanisms behind the noise suppression is of great interest for physicists and electronic engineers. However, the experimental evidence of such shot noise suppression mechanisms is rather difficult. In this work we present the characterization of recessed planar InGaAs/InAlAs diodes with lengths in the order of hundreds of nm. The aim of the study is to identify potential evidences of suppressed shot noise in the structure.

\section{MONTE CARLO ANALYSIS}

In a first step, the devices have been analyzed using a simulator based on the Monte Carlo particle method. This tool provides valuable information about the carrier transport and electrical features along the structure that cannot be easily extracted from experimental measurements. The geometry of the diode is depicted in Fig. 1 and basically consists in an ungated HEMT topology with a recess between the drain and source terminals [4]. Removing the upper InGaAs highly doped layer (cap) in a given region focuses the potential drop and the electric field in that section, which, combined with the high mobility of the channel material, allows expecting quasiballistic motion of carriers under the recess even at room temperature.

The simulated potential profile for a device with dimensions $L_{\mathrm{S}}=200 \mathrm{~nm}, L_{\mathrm{R}}=160 \mathrm{~nm}$ and $L_{\mathrm{D}}=300 \mathrm{~nm}$ is represented in Fig. 2. As it can be observed in the plot, there is a barrier in the potential profile under the source side of the recess, which, by modulating the passage of ballistic carriers, is expected to suppress the associated, shot noise by virtue of Coulomb correlations [2]. The barrier reduces its height with increasing applied voltages, but it does not disappear completely according to the simulation, mainly because above $0.7 \mathrm{~V}$ the increment of voltage is absorbed in the drain access region, so that the potential profile in the recess does not change significantly above that value. This threshold voltage coincides with the potential needed to activate the inter-valley mechanisms in the drain section, which in turn makes that zone very resistive for high potentials [4]. Also, it should be noted how the source region has almost no effect in the potential, and therefore in the device performance. A similar behavior has been obtained with devices of different dimensions.

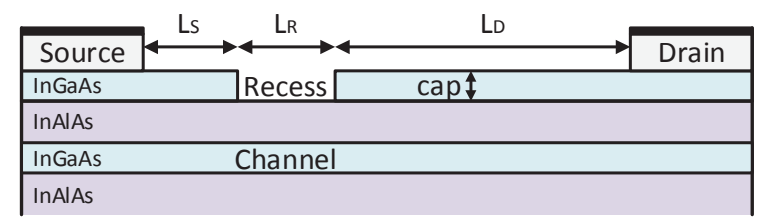

Fig. 1. Sketch of the device structure under analysis. 


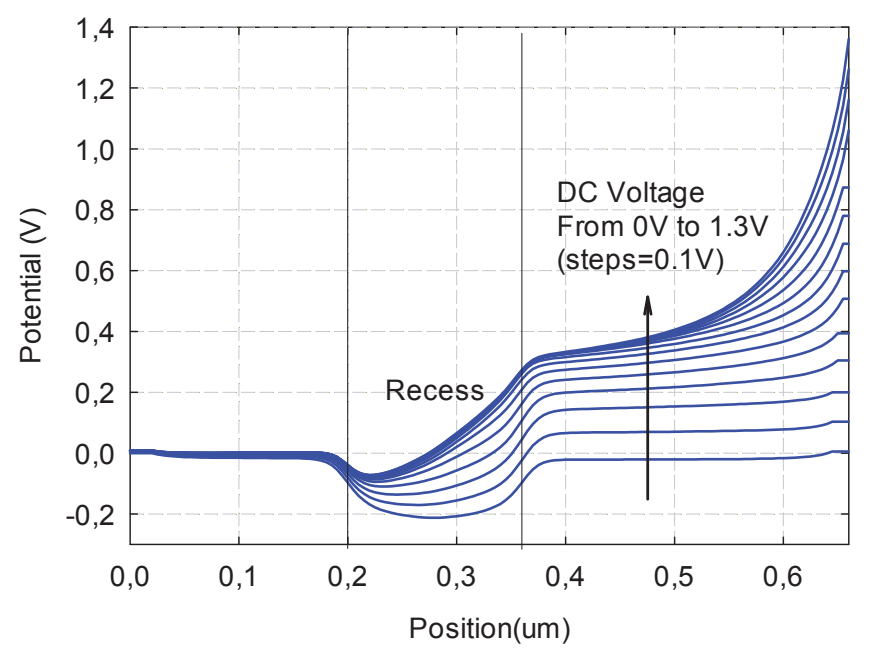

Fig. 2. Simulated potential profile along a structure with $L_{\mathrm{S}}=200 \mathrm{~nm}$, $L_{\mathrm{R}}=160 \mathrm{~nm}$ and $L_{\mathrm{D}}=300 \mathrm{~nm}$.

\section{EXPERIMENTAL RESULTS}

The noise measurements have been performed using a PNA-X N5244A with Option 029 from Agilent Technologies. This instrument allows a simultaneous acquisition of the power density and the reflection coefficient of the device with high levels of sensitivity and accuracy [5]. With both measurements, it is possible to calculate the noise of the device in terms of current spectral density. In this case, the measurements have been obtained averaging in the $20-30 \mathrm{GHz}$ frequency range, ensuring that we are in the plateau beyond $1 / f$ noise or other undesired low-frequency noise effects.

\section{A. Variable Drain Length}

In this first experiment, a set of devices with different drain access lengths, i.e. $L_{\mathrm{D}}=300,550$ and $1000 \mathrm{~nm}$, has been characterized. The recess and source access lengths are $L_{\mathrm{R}}=160 \mathrm{~nm}$ and $L_{\mathrm{S}}=200 \mathrm{~nm}$, respectively. The width of the devices is $10 \mu \mathrm{m}$. For comparison, a device without recess, total length of $1300 \mathrm{~nm}$, and same width has been also measured. The current-voltage curves are shown in Fig. 3. It can be observed that the three devices present similar characteristics, since the current is mainly determined by the recess length, which is a fixed parameter in this case. As expected, the device without recess presents higher levels of current than the recessed samples.

The current spectral densities as a function of the DC current are plotted in Fig. 4. The curve corresponding with full shot noise level, i.e. $S_{\mathrm{I}}=2 q I$, is also represented in the graph for comparison. The device without recess presents a noise density almost flat with current, which may indicate that thermal noise dominates in this case due to the diffusive nature of transport in the device. In the case of the recessed devices, the noise density grows up roughly proportional with current above certain threshold current, but always with levels lower than the full Poissonian value.

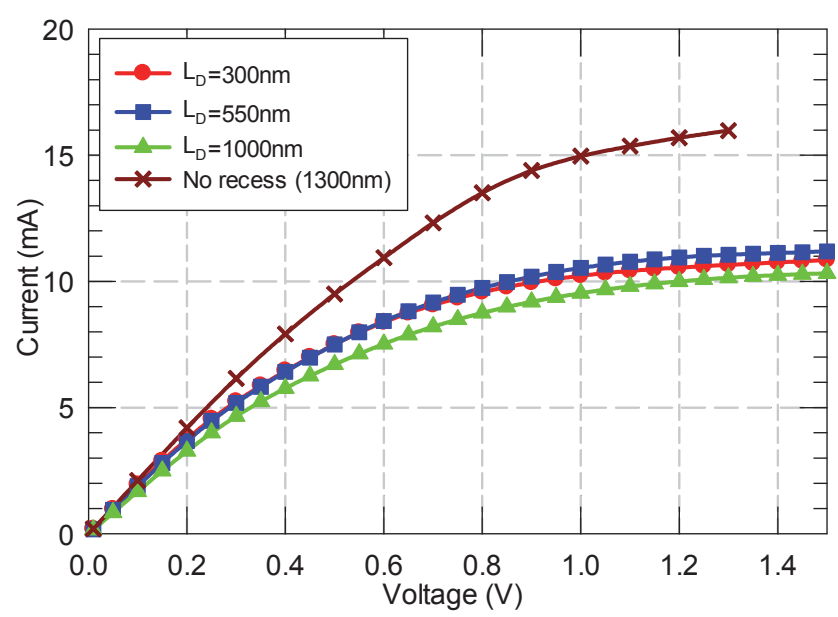

Fig. 3. Measured I-V characteristics of three devices with different drain access lengths $\left(L_{\mathrm{D}}\right)$

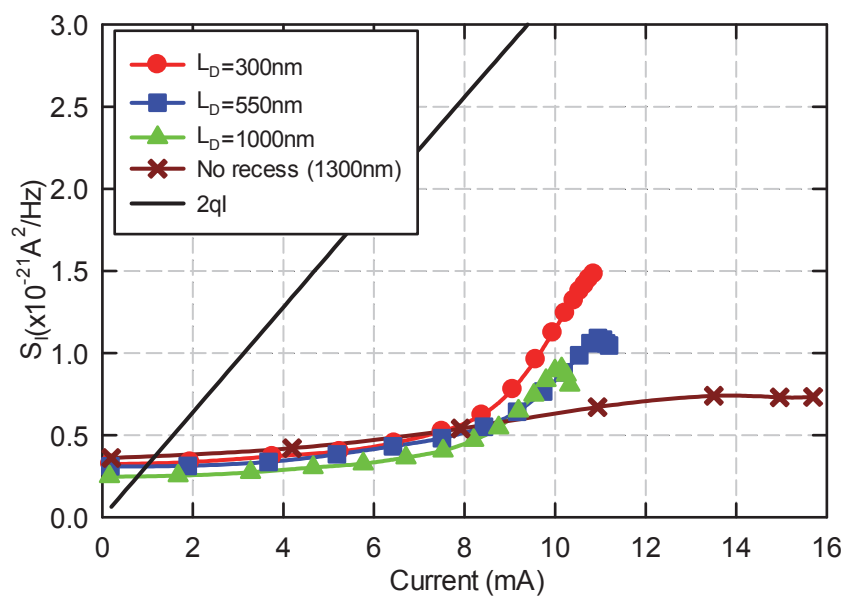

Fig. 4. Measured current spectral densities of three devices with different drain access lengths $\left(L_{\mathrm{D}}\right)$ as a function of the DC current.

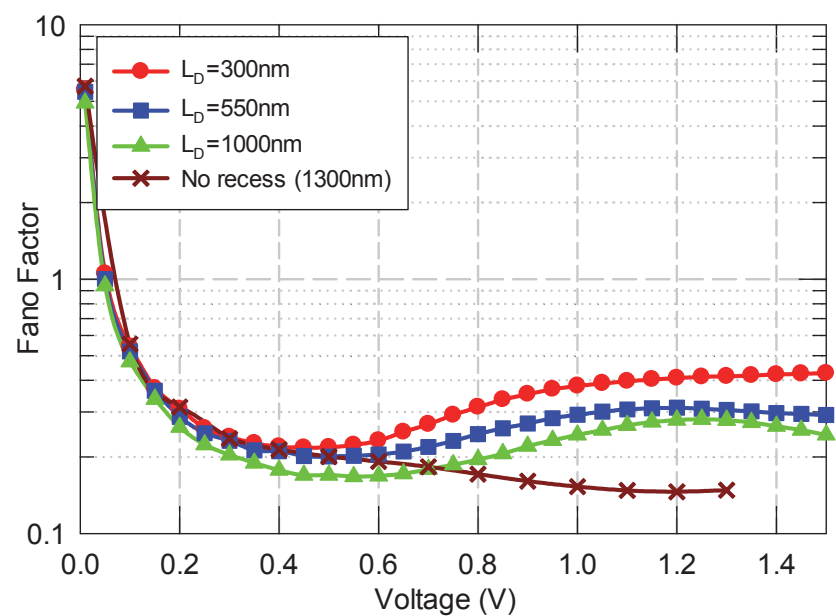

Fig. 5. Measured Fano factor of three devices with different drain access lengths $\left(L_{\mathrm{D}}\right)$ as a function of the DC voltage. 
The representation of the noise response of the three devices in terms of the Fano factor as a function of the bias voltage is shown in Fig. 5. In the case of the device without recess, $F$ is continuously decreasing with voltage. For the recessed devices, $F$ grows up above a given threshold and keeps almost constant for higher potentials. In all the cases the value of $F$ is lower than one in almost all the range of voltages. Furthermore, the value of $F$ appears inversely proportional with $L_{\mathrm{D}}$ at higher voltages. It is precisely for high voltages when we expect the drain region to present high impedance values, so this response can be due to attenuation and/or thermal noise effects in the case of longer drains. Therefore, it is difficult to determine if the observed suppressed noise levels are due to carrier interactions due to the barrier and/or by resistive attenuation in the drain section. Further work is needed to accurately quantify the undesired effects of the drain access and the contact resistance in order to isolate and determine the noise performance in the recess and extract clearer conclusions.

\section{B. Variable Recess Length}

In a second experiment, three devices with different recess lengths, i.e. $L_{\mathrm{R}}=160,400$ and $800 \mathrm{~nm}$, have been characterized. The source and drain accesses are $L_{\mathrm{S}}=200 \mathrm{~nm}$ and $L_{\mathrm{D}}=550 \mathrm{~nm}$ respectively. The width of the devices is $10 \mu \mathrm{m}$. The current-voltage characteristics are shown in Fig. 6. In this case there exist significant differences in the characteristics, since longer recess region makes the device more resistive, and therefore provides lower levels of current. Again, the device without recess of $1300 \mathrm{~nm}$ length and $10 \mu \mathrm{m}$ width is shown for comparison.

The current spectral densities as a function of the DC current are represented in Fig. 7. The line of full shot noise is also plotted for comparison. As in the previous experiment, the current noise curves are flat for low DC currents and they grow up proportional with current above certain threshold. In this case, the Fano factor of the three devices is very similar, as it can be observed in Fig. 8. Again $F$ presents values lower than one in almost all the range of voltages.

\section{CONCLUSION}

The measurements of the current noise density performed in a set of recessed planar InGaAs/InAlAs diodes with different dimensions show potential signs of shot noise suppression in the structures. The appearance of suppressed shot noise in the recess region, where electron transport is quasi ballistic, is attributable to the effect of a fluctuating potential barrier that is able to regulate the electron flow. The presence of such barrier has been evidenced by Monte Carlo simulations of the device structure. However, the simulations also reveal that the drain access region can become very resistive, which would introduce undesired distortions in the overall measurements of the devices. This avoids to precisely extract the value of the noise generated below the recess. Consequently, the quantification of the shot noise suppression exclusively due to carrier interactions induced by the barrier would require a more exhaustive study of all the noise sources involved in the structure in order to isolate the noise generated only in the region of interest.

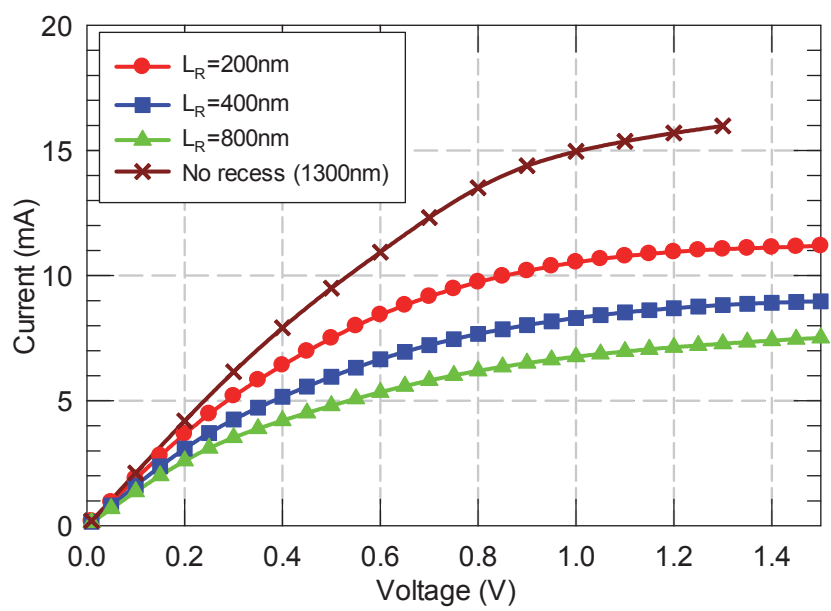

Fig. 6. Measured I-V characteristics of three devices with different recess lengths $\left(L_{\mathrm{R}}\right)$.

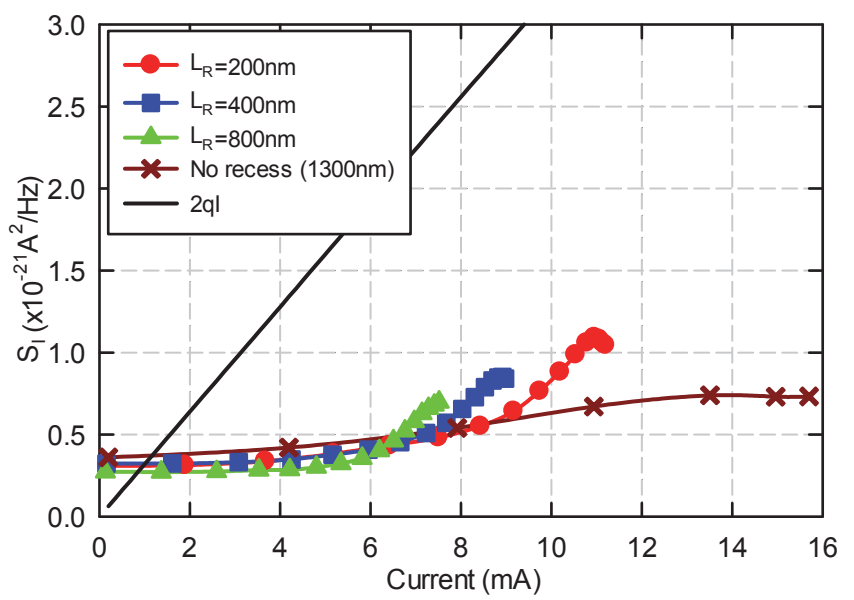

Fig. 7. Measured current spectral densities of three devices with different recess lengths $\left(L_{\mathrm{R}}\right)$ as a function of the DC current.

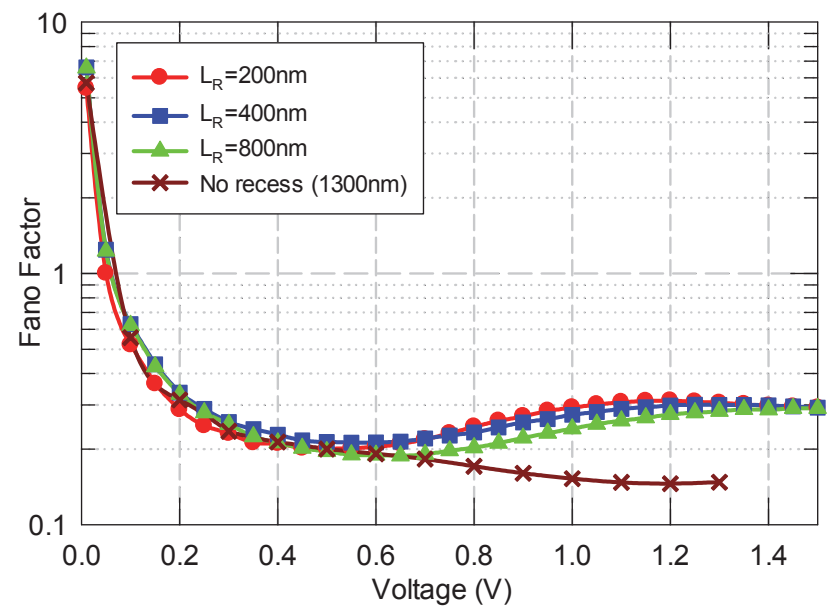

Fig. 8. Measured Fano factor of three devices with different recess lengths $\left(L_{R}\right)$ as a function of the DC voltage. 


\section{ACKNOWLEDGMENT}

This work has been partially supported by the Spanish MINECO through project TEC2013-41640-R, and by the Consejería de la JCyL through project SA052U13. Ó. GarcíaPérez wants to thank the European Social Fund (ESF) for financing his postdoctoral contract.

\section{REFERENCES}

[1] R. Landauer, "The noise is the signal," Nature, vol. 392, pp. 658-659, Apr. 1998.
[2] C. Gomila, I. Cantalapiedra, T. González, L. Reggiani, "Semiclassical theory of shot noise in ballistic $\mathrm{n}^{+}-\mathrm{i}-\mathrm{n}^{+}$semiconductor structures relevance of Pauli and long-range Coulomb correlations," Phys. Rev. B, vol. 66, p. 075302, 2002.

[3] Y. Naveh, A. N. Korotkov, K. K. Likharev, "Shot-noise suppression in multimode ballistic Fermi conductors," Phys. Rev. B, vol. 60, pp. 21692172, Jul. 1999.

[4] S. Pérez, T. González, D. Pardo, J. Mateos, "Terahertz Gunn-like oscillations in InGaAs/InAlAs planar diodes," J. Appl. Phys., vol. 103, p. 094516, 2008.

[5] Agilent Technologies Inc., "High-accuracy noise figure measurements using the PNA-X series network analyzer," Application Note 1408-20 (5990-5800EN), 2013. 